\title{
A NEW WAY FOR TESTING OF LIGHT DEFLECTION IN EARTH ORBIT OR BEYOND
}

\author{
QIN YI-PING \\ Dept. of Astronomy, Beijing Normal University, Bejing, China
}

\begin{abstract}
According to the principles of light deflection, different occulting times of an occultation respond to different basic theories of physics. An occulting way for testing of light deflection in earth orbit or beyond may be possible and is proposed. An application is studied and some suggestions are presented.
\end{abstract}

\section{Introduction}

As is well known, the testing of light deflection is one of the three "classical" tests of relativistic gravity (Will 1981). The conclusion of light deflection can also be derived from other theories, with the results being different from that from general relativity. Generally, the testing of light deflection is operated by observing the deflection angle of light (Will 1981). This kind of operation lasts a long time and brings some observational difficulties.

This work presents an occulting way for testing of light deflection. It avoids the long time observing difficulties.

\section{The Principles and Basic Formulas of Occulting}

Shown in Fig. 1, $P$ presents the occultation while $E$ presents the observing body and $S$ presents the centre of the deflection source. $Q_{1}$ and $Q_{2}$ stand for the two points where the two observed lights pass and the distances to $S$ are the shortest.

Suppose $P$ and $S$ be motionless, and $E$ travel round $S$. When $E$ moves into the opposite side of $S$ relative to $P$ (see Fig. 1), the occulting happens. Let $\alpha$ be the angle, within which $P$ can not be seen by the observer in $E$ (we call that an occulting angle). For a known motion of $E$ traveling round $S$, the occulting angle and occulting time are well related.

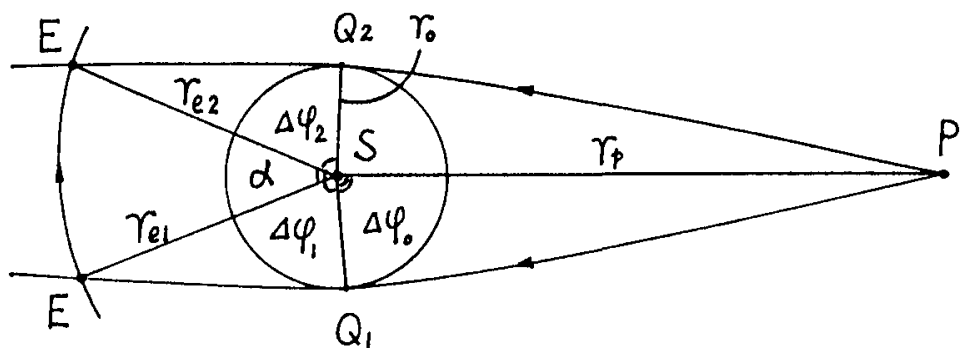

Fig. 1. The diagram of an occultation.

Y. Kondo (ed.), Observatories in Earth Orbit and Beyond, 555-558.

(C) 1990 Kluwer Academic Publishers. Printed in The Netherlands. 
From Fig. 1, we get

$$
\alpha=2 \pi-2 \Delta \varphi_{0}-\Delta \varphi_{1}-\Delta \varphi_{2}
$$

The angles $\Delta \varphi_{0}, \Delta \varphi_{1}$ and $\Delta \varphi_{2}$ can be calculated with the theories of general relativity and Newtonian mechanics. This kind of work is often presented in literature (Carmeli 1982).

\subsection{The angular formula from general RElativity}

In general relativity, the solutions of free fall equations are (Weinberg 1972)

$$
\begin{aligned}
r^{2}(d \varphi / d p) & =J, \\
d t / d p & =b /\left(1-r_{s} / r\right),
\end{aligned}
$$

where $J$ and $b$ are constants, while $r_{s}$ is the Schwarzschild radius, and $p$ is a parameter describing the trajectory.

Applying Eqs. (2) to a photon and using the condition of $(d r / d \varphi)\left(r=r_{0}\right)=0$ (see Fig. 1), we have (for isotropic orbits, it is enough to retain the positive sign)

$$
d \varphi=(1 / r)\left(\left(1-r_{s} / r_{0}\right)\left(r / r_{0}\right)^{2}-1+r_{s} / r\right)^{-\frac{1}{2}} d r .
$$

Expanding Eq. (3) to first order in $r_{s} / r_{0}$, and then integrating it, we have

$$
\begin{aligned}
\varphi\left(r_{2}\right)-\varphi\left(r_{1}\right)= & {\left[-\sin ^{-1}\left(r_{0} / r\right)+(1 / 2)\left(r_{s} / r_{0}\right)\left(1-r_{0}^{2} / r^{2}\right)^{\frac{1}{2}}\right.} \\
& \left.+(1 / 2)\left(r_{s} / r_{0}\right)\left(r-r_{0}\right)^{\frac{1}{2}}\left(r+r_{0}\right)^{-\frac{1}{2}}\right]_{r_{1}}^{r_{2}} .
\end{aligned}
$$

With equation (4), $\Delta \varphi_{0}, \Delta \varphi_{1}$ and $\Delta \varphi_{2}$ in general relativity can be obtained.

\subsection{The angular formula from NeWtonian mechanics}

In Newtonian mechanics, the angular momentum integral and energy conservation equation of two bodies sytem are (Roy 1978)

$$
\begin{aligned}
r^{2}(d \varphi / d t) & =h, \\
(1 / 2) v^{2}-\mu / r & =C,
\end{aligned}
$$

where $h$ and $C$ are constants, while $\mu=G(M=m)$.

Applying Eqs. (5) to a photon and using the conditions $v\left(r=r_{p}\right)=1$ and $(d r / d \varphi)\left(r=r_{0}\right)=0$, we have

$$
\begin{aligned}
d \varphi= & \left(r_{0} / r\right)\left(1+r_{s} / r_{0}-r_{s} / r_{p}\right)^{\frac{1}{2}} \\
& \cdot\left(r^{2}\left(1-r_{s} / r_{p}\right)+r r_{s}-r_{0}^{2}\left(1+r_{s} / r_{0}-r_{s} / r_{p}\right)\right)^{-\frac{1}{2}} d r .
\end{aligned}
$$

Let $k=\left(r_{s} / r_{0}\right) /\left(1+r_{s} / r_{0}-r_{s} / r_{p}\right)$. Expaning Eq. (6) to first order in $k$ and integrating it, we have

$$
\begin{aligned}
\varphi\left(r_{2}\right)-\varphi\left(r_{1}\right)= & {\left[-\sin ^{-1}\left(r_{0} / r\right)\right.} \\
& +(1 / 2)\left(r_{s} / r_{0}\right)\left(1+r_{s} / r_{0}-r_{s} / r_{p}\right)^{-1} \\
& \left.\left(r-r_{0}\right)^{\frac{1}{2}}\left(r+r_{0}\right)^{-\frac{1}{2}}\right]_{r_{1}}^{r_{2}} .
\end{aligned}
$$

With Eq. (7), $\Delta \varphi_{0}, \Delta \varphi_{1}$ and $\Delta \varphi_{2}$ in Newtonian mechanics can be obtained. 


\section{An Application}

Take the sun as $S$ in Fig. 1, and a far distance celestical body as $P$, and the earth as $E$. Considering an average motion, we take $r_{0}=R_{\odot}=6.9599 \cdot 10^{5} \mathrm{~km}$, $r_{e 1}=r_{e 2}=r_{e}=r_{\oplus}=1.4946 \cdot 10^{8} \mathrm{~km}, r_{s}=2.95 \mathrm{~km}, r_{s} / r_{0}=4.239 \cdot 10^{-6}$, $r_{0} / r_{e}=4.656697 \cdot 10^{-3}$.

(i) From Eq. (4), to the accuracy of $10^{-6}$, we then have

$$
\begin{aligned}
& \Delta \varphi_{0}=\pi / 2+r_{s} / r_{0}, \\
& \Delta \varphi_{1}=\Delta \varphi_{2}=\pi / 2-r_{0} / r_{e}+r_{s} / r_{0} .
\end{aligned}
$$

So we get the following from Eq. (1):

$$
\alpha=2 r_{0} / r_{e}-4 r_{s} / r_{0} .
$$

This is the formula of occulting angle in general relativity, where in the right hand side, the first term stands for the motion that a photon travels in a straight line while the second term stands for the correction from general relativity.

(ii) In the same way, Eq. (7) gives (to the accuracy of 10)

$$
\begin{aligned}
\Delta \varphi_{0} & =\pi / 2+(1 / 2) r_{s} / r_{0}, \\
\Delta \varphi_{1} & =\Delta \varphi_{2}=\pi / 2-r_{0} / r_{e}+(1 / 2) r_{s} / r_{0} .
\end{aligned}
$$

So the corresponding occulting angle is

$$
\alpha^{\prime}=2 r_{0} / r_{e}-2 r_{s} / r_{0} .
$$

This is the formula of occulting angle in Newtonian mechanics, where in the right hand side, the second term is the term standing for the correction from Newtonian mechanics.

The time taken by the earth round the sun for a circle is $T=3.1558149984 \cdot 10^{7}$ seconds. Then, the occulting time in general relativity is obtained as

$$
\Delta T=T \alpha /(2 \pi)=(T / \pi)\left(r_{0} / r_{e}-2 r_{s} / r_{0}\right)=12^{h} 58^{m} 12^{s} .66
$$

while the occulting time in Newtonian mechanics is

$$
\Delta T^{\prime}=T \alpha^{\prime} /(2 \pi)=(T / \pi)\left(r_{0} / r_{e}-r_{s} / r_{0}\right)=12^{h} 58^{m} 55^{s} .24
$$

The marks $h, m$ and $s$ denote hours, minutes and seconds.

The difference between the occulting times in Newtonian mechanics and general relativity is

$$
\Delta T^{\prime}-\Delta T=42.58 \text { seconds }
$$

\section{Discussions and Suggestions}

The conclusions of last part show, the occulting time of a star occulted by the sun is about 13 hours. Comparing with the observation of the deflection angle of light, the long time observing difficulties are avoided. It is also shown, the difference of occulting times between the theories of Newtonian mechanics and general relativity 
is about 43 seconds. It is sensitive enough to be detected by instruments if there are no other problems of certainty. We also see that the precise operation of observation is only required at the beginning and the end of an occultation. If an observation is taken in an earth orbit satellite or a vehicle beyond the earth orbit, it occupies only a little time.

On the other side, the observation may produce some difficulties which should be considered seriously. In the following, we present some problems and give some suggestions.

(i) The occulting time involves the geometry of the occulting body. In the case of the sun, if the thickness of the solar chromosphere causes an uncertainty of radius of the sun, the geometrical uncertainty will be in the same order of magnitude of the effect of light deflection. It is depended on the extent of the extinction and the brightness of the solar chromosphere and the magnitude of the observed star, whether the effect of the solar chromosphere should be taken into account.

If the observed star is occulted by the chromosphere, there are some ways tending to solve it. We may use a chromospheric telescope for observing. The distance from the centre of the sun to the edge of the chromosphere is taken as the radius of the occulting body in the theoretical calculation. If the uncertainty of the radius is less than $1 / 16$ of the chromospheric thickness (about 2 seconds of angle), the observation will be valid. Or, when observing an occultation with an ordinary telescope, we observe the chromosphere in the same time. By analysing occulation pictures and the solar chromosphere pictures in different cases, we are supposed to find the effect of the chromosphere because the different thicknesses of the chromosphere may cause different effects (if there is any). With these two kinds of pictures, the radius of the occulting body is also obtained.

(ii) The scattering of rays from atmosphere will strongly effect the optical observation on the ground. To avoid it, a satellite-based observation is needed.

We come to a conclusion that by using an earth orbit or beyond satellite, the observation of an occultation may become a new way for testing of light deflection.

This theory may also be applied to Jupiter or other celestial bodies.

\section{Acknowledgements}

The author thanks Dr. Wu Shi-Min and Dr. Zheng Xue-Tang for all their helps and advices. The author also thanks Dr. Du Sheng-Yun, Dr. Ma Wen-Zhang and Dr. Du Jin-Sheng for their helpful discussions and suggestions.

\section{References}

Carmeli, M.: 1982, Classical Fields, New York: John Wiley and Sons, Inc.2

Roy, A.E.: 1978, Orbital Motion, Bristol: Adam Hilger Ltd)

Weinberg, S.: 1972, Gravitation and Cosmology, (New York: John Wiley and Sons, Inc.

Will, C.M.: 1981a, Theory and Experiment in Gravitational Physics, Cambridge: Cambridge University Press, 166

Will, C.M.: 1981b, Theory and Experiment in Gravitational Physics, Cambridge: Cambridge University Press, 172 